\title{
Programmed cell death may act as a surveillance mechanism to safeguard male gametophyte development in Arabidopsis
}

\author{
Jian Zhang, Chong Teng, Yan Liang ${ }^{\bowtie}$ \\ State Key Laboratory of Plant Genomics and National Plant Gene Research Center (Beijing), Institute of Genetics and \\ Developmental Biology, Chinese Academy of Sciences, Beijing 100101, China \\ $\bowtie$ Correspondence: yliang@genetics.ac.cn \\ Received August 25, 2011 Accepted September 13, 2011
}

\begin{abstract}
Programmed cell death (PCD) plays an important role in plant growth and development as well as in stress responses. During male gametophyte development, it has been proposed that PCD may act as a cellular surveillance mechanism to ensure successful progression of male gametogenesis, and this suicide protective machinery is repressed under favorable growth conditions. However, the regulatory mechanism of male gametophyte-specific PCD remains unknown. Here, we report the use of a TdT-mediated dUTP nick-end labelingbased strategy for genetic screening of Arabidopsis mutants that present PCD phenotype during male gametophyte development. By using this approach, we identified 12 mutants, designated as pcd in male gametogenesis (pig). pig mutants are defective at various stages of male gametophyte development, among which nine pig mutants show a microspore-specific PCD phenotype occurring mainly around pollen mitosis I or the bicellular stage. The PIG1 gene was identified by map-based cloning, and was found to be identical to ATAXIA TELANGIECTASIA MUTATED (ATM), a highly conserved gene in eukaryotes and a key regulator of the DNA damage response. Our results suggest that PCD may act as a general mechanism to safeguard the entire process of male gametophyte development.
\end{abstract}

KEYWORDS programmed cell death, male gametophyte, PIG, TdT-mediated dUTP nick-end labeling, ATM

\section{INTRODUCTION}

In Arabidopsis thaliana, male gametogenesis consists of several distinct stages. In a specialized male reproductive organ called anther, diploid pollen mother cells undergo meiosis and then give rise to tetrads of four haploid microspores. The microspores in the tetrad are released by the action of an enzyme (callase) secreted by the tapedum, the inner layer of the anther. The unicellular microspores then undergo an asymmetric mitotic division known as pollen mitosis I (PM I), resulting in the formation of the bicellular pollen grains consisting of a smaller generative cell and a larger vegetative cell. The smaller generative cell has a condensed nucleus and then undergoes a second pollen mitosis (PM II) to form the two sperm cells, while the larger vegetative cell exits the cell cycle. The Arabidopsis pollen grains contain three cells at the tricellular stage: two sperm cells and one vegetative cell which eventually will form the pollen tube. Male gametogenesis is completed when the sperm cells are released from the anther (McCormick, 1993; Sanders et al., 1999; McCormick, 2004; Ma, 2005).

Programmed cell death (PCD) is a genetically regulated process by which unwanted cells deliberately suicide themselves (Lockshin, 1969; Lockshin and Zakeri, 2004). The common phenotype of PCD contains membrane blebbing, cytoplasmic shrinkage, nuclear condensation and DNA fragmentation (Bursch et al., 2000). TUNEL (TdT-mediated dUTP nick-end labeling) method, using TdT (terminal deoxynucleotidyl transferase) to label 3'-OH groups during DNA fragmentation, is considered as a good specific method to detect cell death by PCD (Gavrieli et al., 1992). In plants, PCD plays an important role in not only the response to the biotic or antibiotic stress, but also the developmental processes including leaf senescence, xylem development, the removing of root cap cells and disappearance of aleurone cells (Pennell and Lamb, 1997; Kuriyama and Fukuda, 2002). 
In addition, PCD has been proposed to act as a cellular surveillance mechanism to safeguard the meiosis and the second mitosis progression of male gametophyte development (Yang et al., 2003; Teng et al., 2008). Under normal growth condition, MMD1 (Male Meiocyte Death1), encoding a PHD-finger protein, may ensure the male gametophyte development by repressing a meiocytic cell death pathway. Mutation of MMD1 causing the meiosis defection directly or indirectly triggers a cell death pathway (Yang et al., 2003). Similarly, mutation in FBR11 (Fumonisin $B_{1}$ Resistant11), encoding a rate-limiting enzyme in de novo sphingolipid synthesis, causes cellular disruption and PCD features during the second mitosis of male gametophyte development. However, it is still unclear whether a similar mechanism occurs at the first mitosis stage of male gametophyte development, and whether other genes are involved in the regulation of male gametophyte-specific PCD. In this study, we identified 12 pig ( $p c d$ in male gametogenesis) mutants all exhibiting PCD phenotype detected by TUNEL analysis, but they are defective at various stages of male gametophyte development including the first mitosis stage. These results suggest that PCD may act as a common cellular surveillance mechanism to safeguard the entire process of male gametophyte development.

\section{RESULTS}

\section{Identification of pcd in male gametogenesis (pig) mutants}

Previous studies revealed that PCD might play an important role in the regulation of male gametophyte development (Yang et al., 2003; Teng et al., 2008). To explore whether PCD is a general regulatory mechanism in male gametophyte development, we performed a genetic screen for mutants that show PCD phenotype during male gametogenesis (Fig. 1). To this end, we first identified mutants with defective gametogenesis by screening a T-DNA mutagenized population showing a distorted segregation ratio for the kanamycin resistance phenotype conferred by the T-DNA insertion. Because kanamycin-resistance is a dominant trait, the phenotype should be segregated in a typical Mendelian pattern of 1:3 (sensitive to resistant). However, a gametophytic mutation caused by a single T-DNA insertion, either female- or male-specific, will alter the segregation ratio to 1:1. This distorted segregation ratio has allowed the identification of many gametophytic mutations from foreign DNA insertional mutants (Howden et al., 1998; Shi et al., 2005). Based on this strategy, we identified approximately 350 putative mutants with a segregation ratio of 1:1 (kanamycin sensitive: resistant) by screening over 20,000 transgenic T2 families (Fig. 1).

Because putative gametophytic mutants identified by this method may be at least both female- or male-gametophytespecific, we therefore analyzed pollen development of these

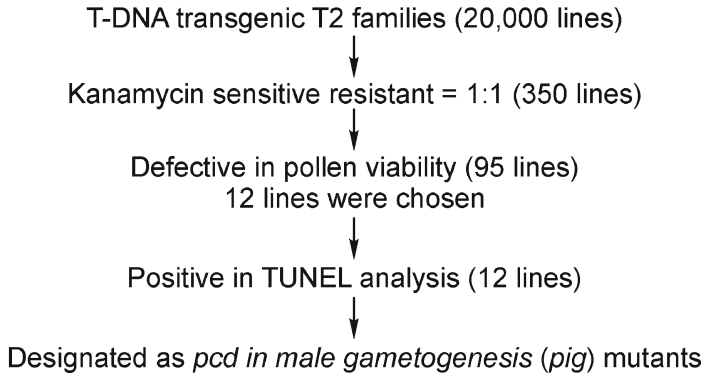

Figure 1. The flow chart of the genetic screen of pcd in male gametogenesis (pig) mutants.

putative mutants by Alexander staining. After the staining, normal pollens exhibited dark-brown color, whereas abnormal or dead pollens showed green color (Fig. 2A). Among the 350 putative mutants, we identified 95 lines, of which more than half of the examined pollens stained with green color (see representative examples in Fig. $2 \mathrm{~B}$ and $2 \mathrm{C}$ ). Of these mutants, several lines showed extremely severe phenotype with only a few viable pollen grains (Fig. 2B).

For this study, we selected 12 mutant lines for further characterization. To rule out the possibility that PCD phenotype is correlated with the severity of the pollen defection, we chose 12 mutants containing both severe like pig1 and non-severe like pig4 phenotype based on Alexander staining. Data presented below demonstrated that these mutants showed PCD phenotype during male gametophyte development, and we therefore designated these mutants as pig1, pig2...to pig12.

\section{Genetic analysis of the pig mutants}

The putative pig mutants were initially identified by the distorted segregation ratio of kanamycin-resistance, which may not co-segregate with the pollen abortive phenotype. Therefore, we examined the genetic nature of these 12 putative mutants by analyzing pollen development using the Alexander staining method. Pollens were collected from progenies derived from self-pollinated putative pig/+ heterozygous plants, and then subjected to Alexander staining. Among the 12 putative pig mutant plants, progenies derived from self-pollinated pig1/+ and pig2/+ plants showed a 3:1 ratio with the pollen development phenotype, i.e., $75 \%$ progenies showed normal pollen development, whereas $25 \%$ progenies showed defective pollen development. This result suggests that pig1 and pig2 are defective in sporophytic development, which consequently causes defective pollen development (see also below).

The other ten mutants (pig3-pig12) showed a 1:1 segregation ratio, similar to that observed in the analysis of kanamycin-resistance. In these ten mutants, approximately $50 \%$ progenies produced normal pollens, whereas the remaining half of the progenies had both normal and aborted 

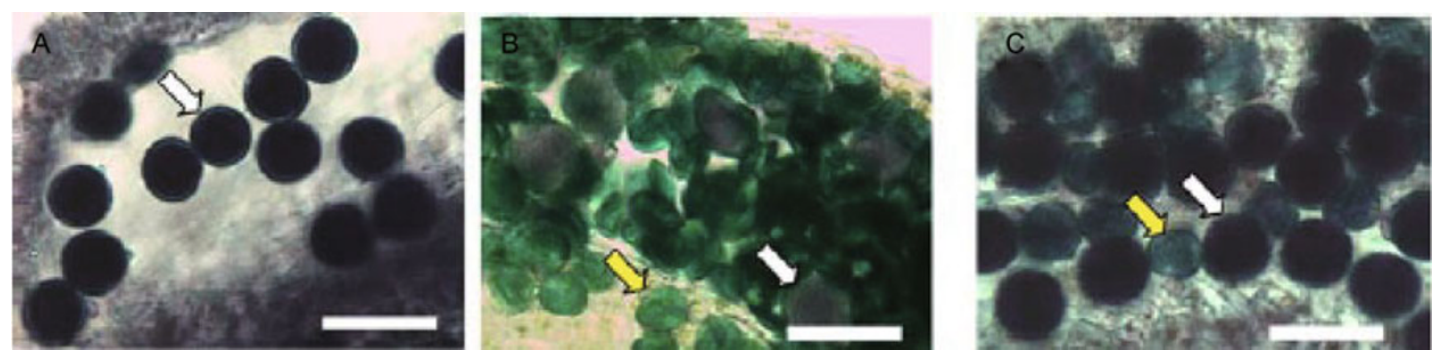

Figure 2. Pollen viability determined by Alexander staining. (A) Mature pollen grains from wild type plants; (B) Mature pollen grains from pig1 mutants; $(C)$ Mature pollen grains from pig4 mutants. The viable pollens are stained dark-brown (white arrow), whereas the unviable pollens are stained green (yellow arrow). Bars, $50 \mu \mathrm{m}$.

pollens with approximately $50 \%$ each. These results indicate that pig3-pig12 are male gametophytic mutants.

\section{Analysis of DNA fragmentation in male gametophytes of the pig mutants}

DNA fragmentation is a key cellular feature of PCD, which produces a free $3^{\prime}-\mathrm{OH}$ group (Williams et al., 1974; Wyllie, 1980). The free 3 '-OH group can be detected by the TdTmediated dUTP nick-end labeling (TUNEL) method to assay possible DNA fragmentation in a cell (Gavrieli et al., 1992). To ask whether pollen abortion in the putative pig mutants is related to PCD, we performed a whole-mount TUNEL analysis in preparations containing male gametophytes (Teng et al., 2008). Flowers at developmental stages 1-6 (the developmental stages were defined according to (Lalanne and Twell, 2002)) were collected, which contained male gametophytes at the unicellular, bicellular and tricellular stages (Lalanne and Twell, 2002). In the preparations made from wild-type flowers, although non-specific signals were often observed at the cell wall of pollen grains, no fluorescent signal was detected in the nuclei of the pollen grain at various stages (Fig. 3A-C). In contrast to that observed in wild type pollen grains, positive TUNEL signals were observed in the nuclei of the pollen produced by all 12 mutants (Fig. 3D, 3E and Fig. S1). These results indicated that all these $12 \mathrm{pig}$ mutants showed PCD phenotype.

\section{The pig mutants are defective at different stages of male gametogenesis}

Data presented above suggest that mutations in the PIG genes cause PCD in male gametophytes. In particular, the pig1 and pig2 mutations resulted in sporophytic defects. On the other hand, pig3-pig12 mutants showed male gametophyte-specific defects. Since TUNEL analysis was performed in a group of flowers with stages 1 to 6 , we could not distinguish the defective stages in mutant pollens. Therefore, we examined the defective stages in 10 mutants of

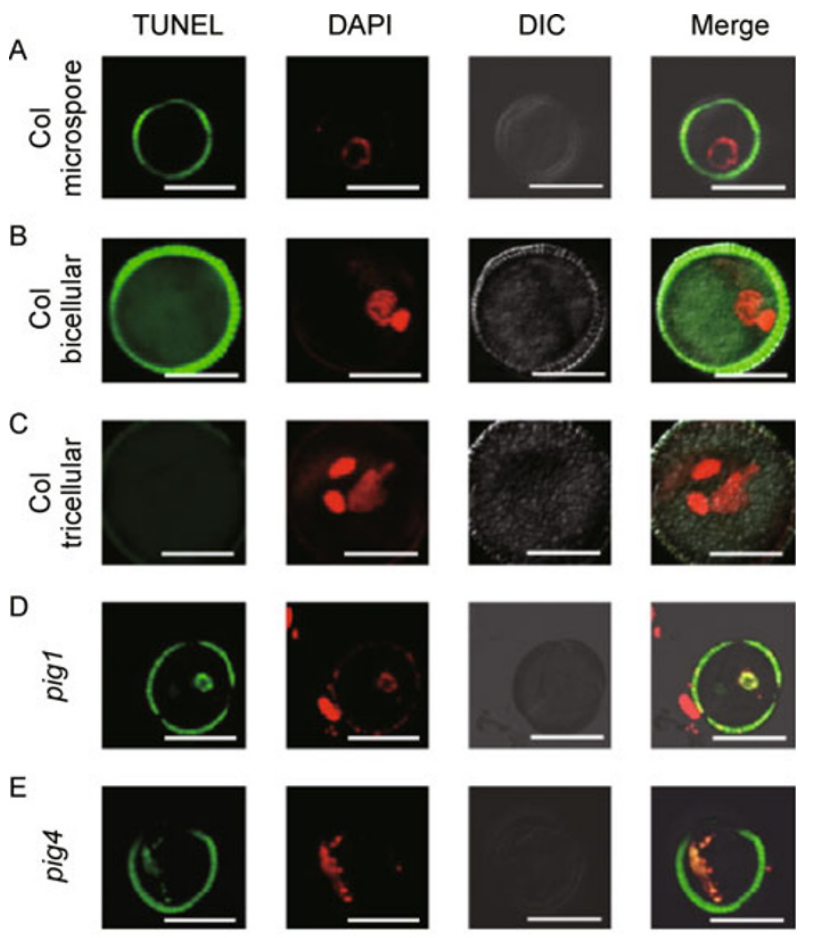

Figure 3. DNA fragmentation detected by TUNEL analysis in pig1 and pig4 mutants. (A) Unicellular stage of wild type pollen grains; (B) Bicellular stage of wild type pollen grains; (C) Tricellular stage of wild type pollen grains; (D) Pollen grains from pig1 mutants; (E) Pollen grains from pig4 mutants. Fluorescence signal after TUNEL analysis is shown in the left panel. DAPI and phase images (DIC) of the corresponding fields are shown in the middle panels as indicated. The right panel shows the merged images of the above channels. Bars, $10 \mu \mathrm{m}$.

pig3-pig12 by DAPI (4',6-diamidino-2-phenylindole) staining, a fluorescent dye specifically binding to DNA and labeling the nucleus. In the ten pig mutants, no abnormality was observed at the unicellular stage (representative example from pig4 is 
shown in Fig. 4A). However, nine mutants from pig3 to pig11 showed apparent defects at the bicellular stage, as indicated by diffusely stained nuclei (representative example from pig 4 is shown in Fig. 4B). These observations indicate that the pig3-pig11 mutants are defective at PM I stage. The pig12 mutants did not have apparent defects at the bicellular stage (Fig. 4C), but were abnormal at the tricellular stage with smallsized or empty pollen grains without visible nuclei (Fig. 4D). This result suggests that pig12 is defective at PM II stage. Our results suggest that microspore-specific PCD phenotype occurs mainly around PM I or the bicellular stages.
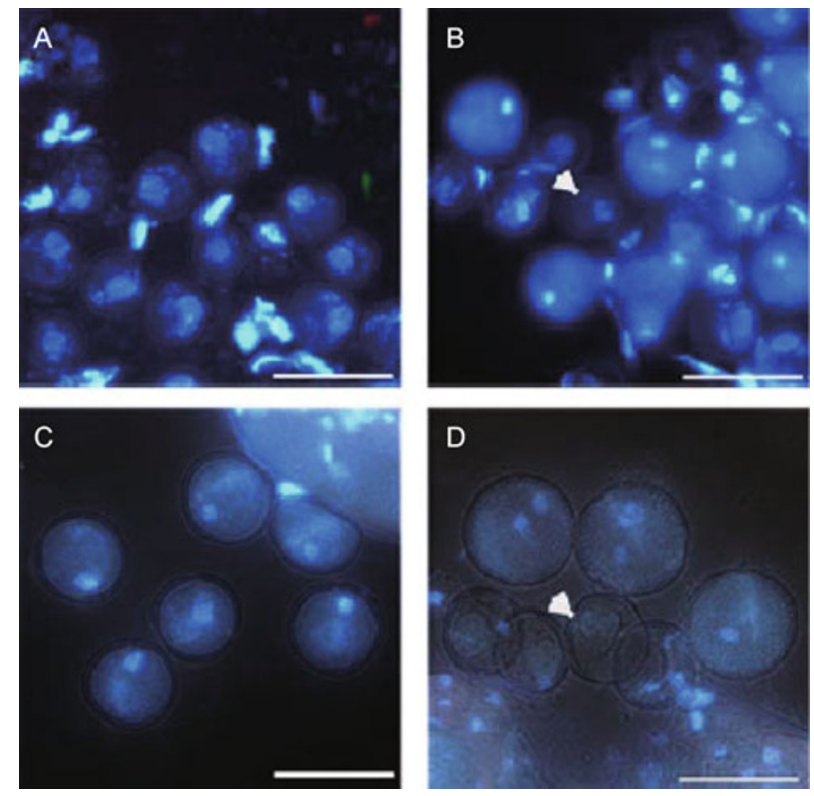

Figure 4. Stages of pollen grain abortion examined by DAPI staining. (A) The microspore stage of pollen grains from pig4 mutants. All pollens show normal DAPI staining. (B) The bicellular stage of pollen grains from pig 4 mutants. Normal pollens are stained as single bright generative nucleus, whereas abnormal pollens show diffusely stained nucleus as arrow indicated. (C) The bicellular stage of pollen grains from pig12 mutants. All pollens are stained normally with single bright generative nucleus. (D) The tricellular stage of pollen grains from pig12 mutants. Normal pollens are stained as two bright generative nuclei, whereas abnormal pollens are empty without visible nuclei as arrow indicated. Bars, $50 \mu \mathrm{m}$.

To better understand the microspore-specific PCD at PM I stage, we examined other PCD features in pig3 mutants. Semi-thin sections were performed from anthers collected at various developmental stages of wild-type and pig 3 mutants, and then analyzed by light microscopy. No abnormality was observed at unicellular stage, whereas small and misshaped pollens were observed at bicellular and tricellular stages (Fig. 5A-C). To obtain the nuclear morphology, the section samples were analyzed by fluorescence microscopy after
DAPI staining. At unicellular stage, all microspores from pig3 mutants showed one nucleus and normal speckled cytoplasm because of the staining of the mitochondrial and plastid DNA (Fig. 5D and 5G) (Regan and Moffatt, 1990). However, at bicellular and tricellular stages, only half of pollens showed wild type brightly stained generative nuclei, whereas half of pollens showed abnormal nuclei with diffusely DAPI staining (Fig. 5E and 5F). In comparison with the wild type pollen (Fig. 5H and 5I), PCD features including cytoplasmic shrinkage and nuclear degeneration were observed at bicellular and tricellular stages in the mutant pollen (Fig. $5 \mathrm{~J}$ and $5 \mathrm{~K}$ ). Therefore, our further analysis confirmed that PCD is present at first mitosis stage and might act as a cellular surveillance mechanism to ensure the successful complementation of this progression.

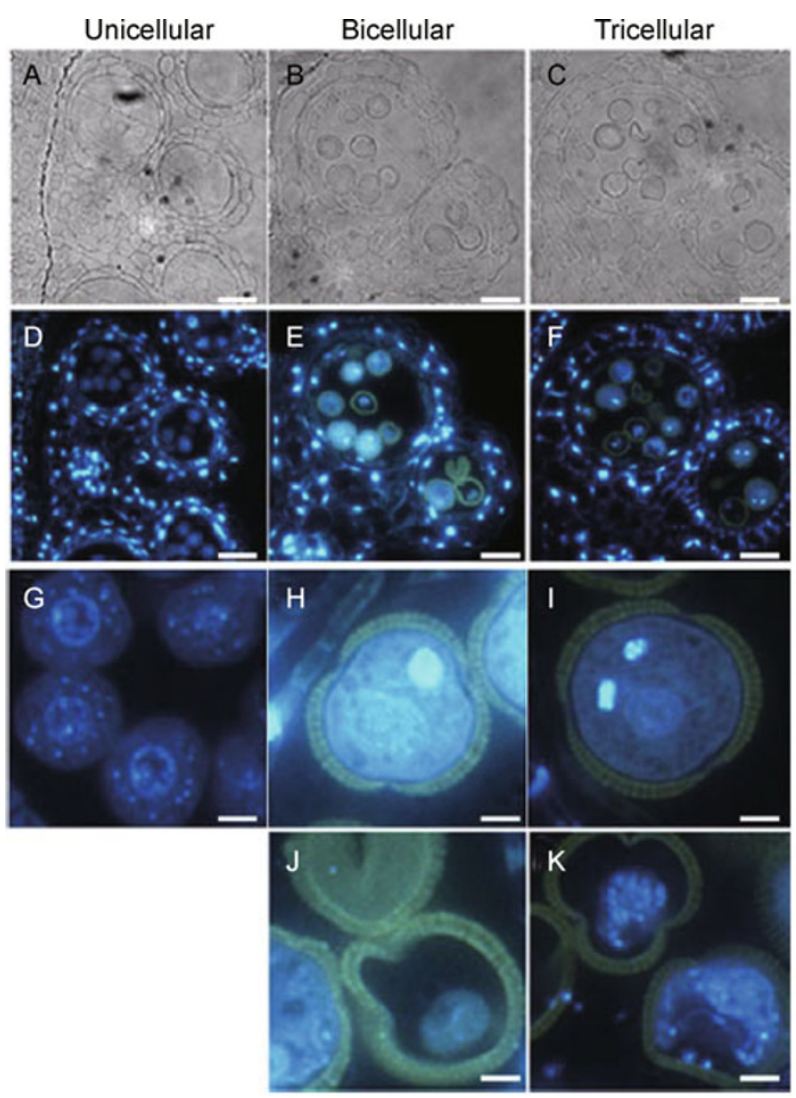

Figure 5. Developmental defects of pig3 mutants during male gametogenesis. (A-C) Semi-thin section of pig3 anthers under a light microscope; (D-F) Fluorescence observation of $(A-C)$ after DAPI staining; (G-K) Magnified pictures of (D-F); $(\mathrm{G}-\mathrm{I})$ showing the wild type pollen grains, while $(\mathrm{J})$ and $(\mathrm{K})$ showing the defective pollen grains. Developmental stages of male gametophyte are showed on the top of the figures. Bars for $(A-F), 50 \mu \mathrm{m}$; Bars for (G-K), $10 \mu \mathrm{m}$.

Taken together, these results indicate that defective male gametophyte development occurs at all major developmental 
stages of the pig mutants, including meiosis, PM I and PM II, with a relatively higher frequency at PM I.

\section{PIG1 is allelic to ATAXIA TELANGIECTASIA MUTATED (ATM)}

To get more insight into the molecular mechanism of PIGregulated male gametophyte development, we attempted to identify and characterize representative PIG genes. Results obtained from partial characterization of PIG1 are reported below.

The pollen defective phenotype of pig1 mutants is constant after the backcross. In an F1 population obtained from the backcross, the progenies showed a wild-type phenotype. In F2 plants, approximately $25 \%$ progenies showed a pig1 phenotype, characteristics of a Mendelian segregation pattern (pig1: wild-type $=14: 48, x^{2}=0.38$ ). This result indicates that $P I G 1$ mutation is recessive in a single nuclear gene. Subsequent studies indicated that the pig1 mutation was not tagged by the T-DNA insert. Therefore, we performed a positional cloning experiment to identify the PIG1 gene. A genetic mapping population was generated by crossing pig1 (Col-0) with wild type Ler plants. Using an F2 population of 133 pig1 mutant plants, PIG1 was mapped onto chromosome III between two SSLP markers T17F15 and T24C20.2, in an interval of $169 \mathrm{~Kb}$ (Fig. 6A). This region contained 37 annotated genes. DNA sequencing analysis of the pig1 genome revealed a large fragment of $13,071 \mathrm{bp}$ in the ATAXIA TELANGIECTASIA MUTATED (ATM) gene was deleted and replaced by a small fragment of filler DNA (Fig. 6B). Consistent with the mutation nature of pig1 in the ATM gene, reverse transcription (RT)-PCR analysis revealed that no transcript was detected in the deleted region of ATM. However, an ATM transcript was detected using primer pairs upstream of the deletion region (Fig. $6 \mathrm{C}$ and $6 \mathrm{D}$ ), suggesting that PIG1 mutation most likely causes the generation of a truncated transcript of ATM.

The pig1 mutant shows a similar phenotype as several atm mutants previously characterized (Garcia et al., 2003). To verify whether the pig1 mutant phenotype is caused by the deletional mutation in ATM, we performed an allelism analysis of pig1 with atm-2 (Garcia et al., 2003). From a cross between pig1/+ (female) and atm-2/+ (male), two pig1/atm-2 plants were identified out of $13 \mathrm{~F} 1$ progenies assessed by phenotyping and genotyping. Pollen grains produced from pig1/atm-2 plants were stained as green by Alexander's staining solution (Fig. 7A and 7B). The siliques of pig1/atm-2 were shorter than that of wild type (Fig. 7C and 7D). These results demonstrate that the allelism of pig1 and atm-2, and that the pig1 mutant phenotype are caused by the mutation in ATM gene.

\section{DISCUSSION}

Male gametogenesis is an essential process for the formation

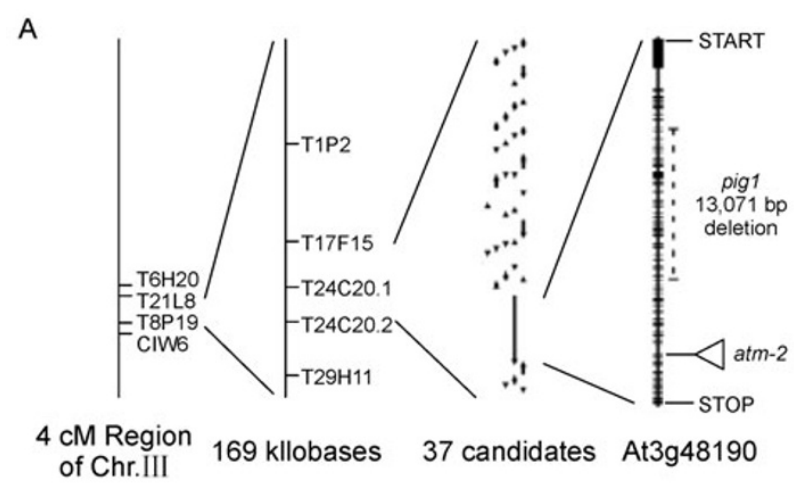

B

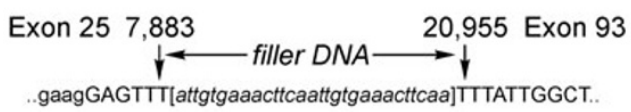

C

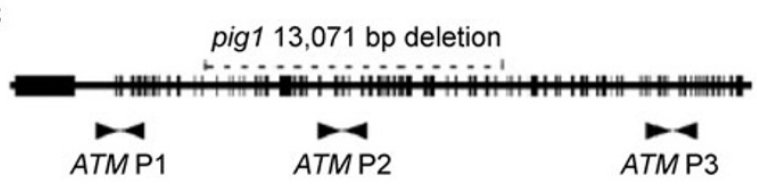

D

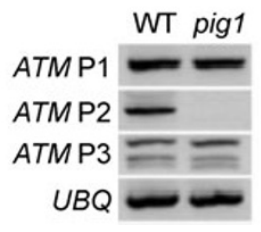

Figure 6. Molecular characterization of the PIG1 gene. (A) The genetic and physical map of PIG1 gene. The PIG1 locus was mapped to a $4 \mathrm{cM}$ region with flanking markers $\mathrm{T} 6 \mathrm{H} 2 \mathrm{O}$ and CIW6 on chromosome III. Subsequent mapping with 133 pig1 homozygotes showed that PIG1 was mapped to a $169 \mathrm{~kb}$ region with flanking markers T17F15 and T24C20.2. Thirty seven candidate genes were identified in that region according to the Col-0 sequence in GenBank. Directly sequencing indicated that a 13,071 bp DNA deletion in the open reading frame (ORF) of ATAXIA TELANGIECTASIA MUTATED (ATM, At3g48190) may cause the mutation of PIG1 gene. Filled boxes represent the predicted exons of the PIG1/ATM gene. The dot line indicates the missing region in pig1 mutants. The triangle represents the T-DNA insertion site in atm-2 mutants. (B) The filler DNA sequence in pig1 mutants. Number above indicates the position in wild type PIG1. The filler DNA sequence found in the pig1 mutants are indicated in lowercase italic within the square brackets. (C) Schematic diagram of ATM gene. Filled boxes represent the predicted exons of the PIG1/ATM gene. The dot line indicates the deleted sequences in pig1 mutants. (D) ATM/PIG1 expression was analyzed by semi-quantitative PCR in pig1 and wild type. Ubiquitin (UBQ) was used as an internal control, and the position of primer pairs are indicated in (C). 

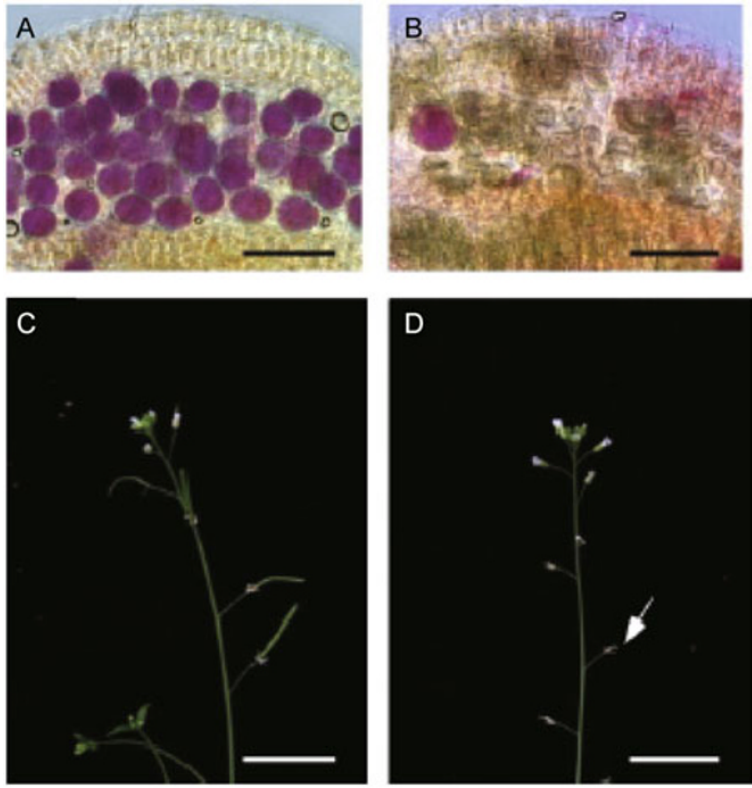

Figure 7. Phenotype analysis of pig1atm-2 double mutants. (A) Alexander's test of mature pollen grains from wild type; (B) Alexander's test of mature pollen grains from pig1atm-2 double mutants; (C) Flowering stems of wild type (6-week-old); (D) Flowering stems of pig1atm-2 double mutants (6-week-old). Siliques of pig1atm-2 double mutants are shorter than that of the corresponding wild type as arrow indicated. Bars for $A$ and $B, 50 \mu \mathrm{m}$. Bars for $C$ and $D, 2 \mathrm{~cm}$.

mechanisms including PCD (Bakeeva et al., 2005). During male gametophyte development, the tupedum, the endothecium and the epidermis all undergo PCD, eventually leading to the release of pollen grains (Papini et al., 1999; Varnier et al., 2005; Li et al., 2006; Vizcay-Barrena and Wilson, 2006). In those cases, PCD is a developmental program to eliminate the unwanted cells, but not triggered by the extensive damage of male gametophyte. The recent studies of $\mathrm{mmd} 1$ and fbr11 mutants in Arabidopsis indicated that PCD could act as a surveillance mechanism to remove the damaged cells at meiosis and PM II stages during male gametophyte development (Yang et al., 2003; Teng et al., 2008). In this study, we isolated 12 pig mutants which all showed PCD phenotype detected by TUNEL analysis, but were defective at various stages of male gametophyte development including the PM I stage. Our results suggest that PCD is a general surveillance mechanism for monitoring the entire development of male gametophyte. However, except MMD1 and FBR11, the molecular study of this mechanism is still in its infancy. MMD1 gene encodes a PHD-finger protein probably associated with chromatin-remodeling, and its mutation results in meiotic defect and apoptosis-like phenotype, suggesting that MMD1 may regulate the male gametophyte-specific PCD at meiosis stage (Yang et al., 2003). FBR11 encodes a key enzyme for the synthesis of sphingolipid which is an important signal for cell death. The cellular defection and PCD features observed at the PM II stage of the male gametophyte development in fbr11 mutants suggest that sphingolipid may be involved in the male gametophyte-specific PCD during the second mitosis (Teng et al., 2008). Therefore, in addition to MMD1 and FBR11, further studies of pig mutants might provide new insight into the molecular mechanism of the male gametophyte-specific PCD in pollen development.

ATM (Ataxia-telangiectasia mutated) gene is a key factor in damage response to DNA double-strand breaks, first reported in ataxia-telangiectasia disease (Boder and Sedgwick, 1958; Centerwall and Miller, 1958). Studies of ATM genes in yeast, fly and mice indicated that ATM gene is not only important for the response to DNA damage, but also essential for meiosis (Carpenter, 1979; Kato and Ogawa, 1994; Lydall et al., 1996). Similarly, Arabidopsis atm mutants are hyposensitive to some DNA-damaging treatments and partially sterile due to the extensive chromosome fragmentation in meiocytes (Garcia et al., 2003). It has been reported that DNA damage-induced apoptosis is a common surveillance mechanism to maintain genomic stability in many organisms (Gartner et al., 2000; Norbury and Zhivotovsky, 2004). In mice, atm-deficiency causes meiosis arrest during male gametogenesis followed by apoptotic degeneration (Barlow et al., 1997). Here, we isolated pig1 mutants, an allele of Arabidopsis atm, showing PCD phenotype during male gametophyte development. Combined with the meiosis defects in atm mutants with PCD feature in pig1 mutants, ATMIPIG1 might regulate the DNA damage-induced apoptosis during male gametophyte development.

In summary, we isolated 12 pig mutants which are defective at various stages of male gametophyte development, exhibiting the male gametophyte-specific PCD features. This study demonstrate that PCD may play a surveillance role during the entire process of male gametophyte development.

\section{MATERIALS AND METHODS}

Plant materials, growth conditions and genetic screening

Unless otherwise indicated, the Columbia-0 (Col-0) ecotype of Arabidopsis thaliana (L.) Heynh. was used in this study. atm-2 mutants (SALK_006953) were obtained from the Arabidopsis Biological Resources Center (ABRC).

The genetic screen was performed in a T-DNA mutagenized population which is generated by a pER16 vector containing kanamycin resistance gene (Zuo et al., 2000). Approximately 20,000 independent T2 families were screened for male gametophytic mutants on the basis of their distorted segregation ratios of kanamycin resistance. Seeds were surface-sterilized in $10 \%$ sodium hypochlorite for $10 \mathrm{~min}$, washed thoroughly with sterile water and then evenly distributed on plates containing $1 / 2$ Murashige-Skoog (MS) agar (1/2 $\times$ MS salts, $1 \%$ sucrose, $0.8 \%$ agar) supplemented with 50 $\mathrm{mg} / \mathrm{mL}$ kanamycin (Murashige and Skoog, 1962). Plates were sealed with surgical type, placed at $4^{\circ} \mathrm{C}$ in the dark for two days, and then 
transferred to continuous fluorescent white light. After two weeks, the kanamycin phenotype (resistance or sensitive) was scored and kanamycin resistance seedlings were transferred to pots containing vermiculite saturated with Gamborg's B5 liquid medium. Plants were grown under a $16 \mathrm{~h}$ light $/ 8 \mathrm{~h}$ dark cycle at $22^{\circ} \mathrm{C}$ and seeds were collected from T2 individuals.

Reciprocal crosses and genetic analysis of male gametophytic mutants were performed essentially as previously described (Howden et al., 1998).

\section{Alexander test and DAPI (4',6-diamidino-2-phenylindole) staining}

Alexander staining was performed as previously described (Alexander, 1969). Released pollen grains were directly immersed in Alexander solution, and then were observed under a light microscope (Olympus BX51). DAPI staining was carried out following the procedures described in (Regan and Moffatt, 1990). For wholemount DAPI staining, pollen nuclei were stained with $1 \mathrm{mg} / \mathrm{mL}$ DAPI for $5 \mathrm{~min}$ at room temperature, and then mounted temporarily on a slide with $50 \%$ glycerol in PBS (phosphate-buffered saline) buffer. Samples for semi-thin section were fixed in formaldehyde acetic acid (FAA) overnight. After dehydration in gradual ethanol series, the samples were embedded in historesin (Leica). Semi-thin sections $(3 \mu \mathrm{m})$ were stained with $1 \mathrm{mg} / \mathrm{mL}$ DAPI for $5 \mathrm{~min}$. Pollen viability after DAPI staining was analyzed under a fluorescent microscope (Olympus BX51).

\section{Whole-mount TUNEL (TdT-Mediated dUTP Nick End Labeling) assay for pollen grains}

For TUNEL assay, pollen grains were fixed in $4 \%$ paraformaldehyde in $0.1 \mathrm{~mol} / \mathrm{L} \mathrm{PBS}(\mathrm{pH} 7.4)$ at $4^{\circ} \mathrm{C}$ overnight. After PBS washing, pollen grains were dehydrated through a graded methanol series $(30 \%$, $50 \%, 70 \%, 100 \%$, and $100 \%$ ) and $100 \%$ xylene with $30 \mathrm{~min}$ incubation for each step, and then rehydrated through a series of methanol $(85 \%, 70 \%, 50 \%, 30 \%, 10 \%$ and water) with $10 \mathrm{~min}$ incubation for each step. Pollen grains were digested with $20 \mu \mathrm{g} / \mathrm{mL}$ proteinase $\mathrm{K}$ at $37^{\circ} \mathrm{C}$ for $35 \mathrm{~min}$ and subsequently washed twice with $2 \mathrm{mg} / \mathrm{mL}$ glycine in PBS. The TUNEL assay was performed using the In Situ Cell Death Detection Kit-Fluorescein (Roche Diagnostics Hong Kong). After TUNEL staining, pollen grains were stained with $1 \mathrm{mg} / \mathrm{mL}$ DAPI for $5 \mathrm{~min}$ at room temperature. Images were taken using a confocal microscope (Olympus FV1000).

\section{Map-based cloning of pig1}

An F2 mapping population was generated by crossing pig1 (Col-0) with wild type Ler plants. The PIG1 gene were mapped by using simple sequence length polymorphisms (SSLP) markers and fine mapped using specific markers (Supplementary Table 1).

\section{RNA isolation and RT-PCR}

Total RNA was extracted from flowers of wild type and pig1 mutants by RNase easy kit (Invitrogen) according to the manufacturer's instructions. RT-PCR was carried out as previously described with modification (Zuo et al., 2000). The reaction was cycled at $94^{\circ} \mathrm{C}$ for $30 \mathrm{~s}, 58^{\circ} \mathrm{C}$ for $30 \mathrm{~s}$, and $72^{\circ} \mathrm{C}$ for $1 \mathrm{~min}, 28-30$ times.

\section{ACKNOWLEDGEMENTS}

This study was performed in the laboratory of Dr. Jianru Zuo, and was supported by a grant from the Ministry of Science and Technology of China (No. 2009CB941502).

Supplementary material is available in the online version of this article at http://dx.doi.org/10.1007/s13238-011-1102-6 and is accessible for authorized users.

\section{REFERENCES}

Alexander, M.P. (1969). Differential staining of aborted and nonaborted pollen. Stain Technol 44, 117-122.

Bakeeva, L.E., Dzyubinskaya, E.V., and Samuilov, V.D. (2005). Programmed cell death in plants: ultrastructural changes in pea guard cells. Biochemistry (Mosc) 70, 972-979.

Barlow, C., Liyanage, M., Moens, P.B., Deng, C.X., Ried, T., and Wynshaw-Boris, A. (1997). Partial rescue of the prophase I defects of Atm-deficient mice by p53 and p21 null alleles. Nat Genet 17, $462-466$.

Boder, E., and Sedgwick, R.P. (1958). Ataxia-telangiectasia; a familial syndrome of progressive cerebellar ataxia, oculocutaneous telangiectasia and frequent pulmonary infection. Pediatrics 21 , 526-554.

Bursch, W., Ellinger, A., Gerner, C., Fröhwein, U., and SchulteHermann, R. (2000). Programmed cell death (PCD). Apoptosis, autophagic PCD, or others? Ann N Y Acad Sci 926, 1-12.

Carpenter, A.T. (1979). Recombination nodules and synaptonemal complex in recombination-defective females of Drosophila melanogaster. Chromosoma 75, 259-292.

Centerwall, W.R., and Miller, M.M. (1958). Ataxia, telangiectasia, and sinopulmonary infections; a syndrome of slowly progressive deterioration in childhood. AMA J Dis Child 95, 385-396.

Garcia, V., Bruchet, H., Camescasse, D., Granier, F., Bouchez, D., and Tissier, A. (2003). AtATM is essential for meiosis and the somatic response to DNA damage in plants. Plant Cell 15, 119-132.

Gartner, A., Milstein, S., Ahmed, S., Hodgkin, J., and Hengartner, M. O. (2000). A conserved checkpoint pathway mediates DNA damage-induced apoptosis and cell cycle arrest in C. elegans. Mol Cell 5, 435-443.

Gavrieli, Y., Sherman, Y., and Ben-Sasson, S.A. (1992). Identification of programmed cell death in situ via specific labeling of nuclear DNA fragmentation. J Cell Biol 119, 493-501.

Howden, R., Park, S.K., Moore, J.M., Orme, J., Grossniklaus, U., and Twell, D. (1998). Selection of T-DNA-tagged male and female gametophytic mutants by segregation distortion in Arabidopsis. Genetics 149, 621-631.

Kato, R., and Ogawa, H. (1994). An essential gene, ESR1, is required for mitotic cell growth, DNA repair and meiotic recombination in Saccharomyces cerevisiae. Nucleic Acids Res 22, 3104-3112.

Kuriyama, H., and Fukuda, H. (2002). Developmental programmed cell death in plants. Curr Opin Plant Biol 5, 568-573.

Lalanne, E., and Twell, D. (2002). Genetic control of male germ unit organization in Arabidopsis. Plant Physiol 129, 865-875. 
Li, N., Zhang, D.S., Liu, H.S., Yin, C.S., Li, X.X., Liang, W.Q., Yuan, Z., $\mathrm{Xu}$, B., Chu, H.W., Wang, J., et al. (2006). The rice tapetum degeneration retardation gene is required for tapetum degradation and anther development. Plant Cell 18, 2999-3014.

Lockshin, R.A. (1969). Programmed cell death. Activation of lysis by a mechanism involving the synthesis of protein. $\mathrm{J}$ Insect Physiol 15, 1505-1516.

Lockshin, R.A., and Zakeri, Z. (2004). When cells die II: a comprehensive evaluation of apoptosis and programmed cell death. Hoboken, N.J.: Wiley-Liss.

Lydall, D., Nikolsky, Y., Bishop, D.K., and Weinert, T. (1996). A meiotic recombination checkpoint controlled by mitotic checkpoint genes. Nature 383, 840-843.

Ma, H. (2005). Molecular genetic analyses of microsporogenesis and microgametogenesis in flowering plants. Annu Rev Plant Biol 56, 393-434.

McCormick, S. (1993). Male gametophyte development. Plant Cell 5, 1265-1275.

McCormick, S. (2004). Control of male gametophyte development. Plant Cell 16, S142-S153.

Murashige, T., and Skoog, F. (1962). A revised medium for rapid growth and bioassays with tobacco tissue cultures. Physiol Plant 15, 473-497.

Norbury, C.J., and Zhivotovsky, B. (2004). DNA damage-induced apoptosis. Oncogene 23, 2797-2808.

Papini, A., Mosti, S., and Brighigna, L. (1999). Programmed-celldeath events during tapetum development of angiosperms. Protoplasma 207, 213-221.

Pennell, R.I., and Lamb, C. (1997). Programmed cell death in plants. Plant Cell 9, 1157-1168.

Regan, S.M., and Moffatt, B.A. (1990). Cytochemical analysis of pollen development in wild-type Arabidopsis and a male-sterile mutant. Plant Cell 2, 877-889.
Sanders, P.M., Bui, A.Q., Weterings, K., Mclntire, K.N., Hsu, Y.-C., Lee, P.Y., Truong, M.T., Beals, T.P., and Goldberg, R.B. (1999). Anther developmental defects in Arabidopsis thaliana male-sterile mutants. Sex Plant Reprod 11, 297-322.

Shi, D.Q., Liu, J., Xiang, Y.H., Ye, D., Sundaresan, V., and Yang, W.C. (2005). SLOW WALKER1, essential for gametogenesis in Arabidopsis, encodes a WD40 protein involved in $18 \mathrm{~S}$ ribosomal RNA biogenesis. Plant Cell 17, 2340-2354.

Teng, C., Dong, H., Shi, L., Deng, Y., Mu, J., Zhang, J., Yang, X., and Zuo, J. (2008). Serine palmitoyltransferase, a key enzyme for de novo synthesis of sphingolipids, is essential for male gametophyte development in Arabidopsis. Plant Physiol 146, 1322-1332.

Varnier, A.L., Mazeyrat-Gourbeyre, F., Sangwan, R.S., and Clément, C. (2005). Programmed cell death progressively models the development of anther sporophytic tissues from the tapetum and is triggered in pollen grains during maturation. J Struct Biol 152, 118-128.

Vizcay-Barrena, G., and Wilson, Z.A. (2006). Altered tapetal PCD and pollen wall development in the Arabidopsis ms1 mutant. J Exp Bot 57, 2709-2717.

Williams, J.R., Little, J.B., and Shipley, W.U. (1974). Association of mammalian cell death with a specific endonucleolytic degradation of DNA. Nature 252, 754-755.

Wyllie, A.H. (1980). Glucocorticoid-induced thymocyte apoptosis is associated with endogenous endonuclease activation. Nature 284, 555-556.

Yang, X., Makaroff, C.A., and Ma, H. (2003). The Arabidopsis MALE MEIOCYTE DEATH1 gene encodes a PHD-finger protein that is required for male meiosis. Plant Cell 15, 1281-1295.

Zuo, J., Niu, Q.W., and Chua, N.H. (2000). Technical advance: An estrogen receptor-based transactivator XVE mediates highly inducible gene expression in transgenic plants. Plant $\mathrm{J} 24$, 265-273. 